\title{
Time-optimal control of a swing
}

\author{
Jayant E. Kulkarni \\ Department of Theoretical and Applied Mechanics \\ Cornell University \\ Ithaca, NY 14850, USA. \\ jayantetam. cornell.edu
}

\begin{abstract}
It is well-known that some form of optimization takes place in skilled human tasks. Using largely numerical techniques researchers in the field of biomechanics have optimized different cost functions to predict human motion for tasks like walking and rowing. In a similar vein in this paper we show, using Pontryagin's Minimum Principle and after some simplification, that the typical pumping strategy used by children on a swing is time-optimal. We present results for the two special cases: When the swing is to be taken from small initial oscillations to a specific final angle in minimum time, and when it is to be brought from an initial angle to rest in minimum time. The method outlined is extendable to other initial and final conditions, though the results obtained for other boundary conditions are not as elegant and intuitive as in the cases discussed.
\end{abstract}

\section{INTRODUCTION}

The idea that skilled human motion entails optimization of some cost function with certain constraints on the control inputs and the phasespace, has become well-entrenched in the field of biomechanics. Several researchers have attempted to predict human motion using the optimization approach for tasks ranging from locomotion to jumping (Bertram and Ruina 2001, Pandy and Anderson 1991). Most of these approaches have been largely numerical in nature. In this paper we obtain analytical results using Pontryagin's Minimum Principle to the time-optimal problem of pumping a swing.

The problem of pumping a swing is a classical one and has been addressed by several researchers from different perspectives. Tea and Falk (1968) show how the elementary concepts of angular momentum and work-energy can be used to explain why the amplitude of a swing increases when it is pumped. Burns (1969) has shown how the problem exhibits a resonance like phenomenon and Wirkus,
Ruina and Rand (1998) compare the two different pumping strategies: When the rider is standing, and when the rider is seated.

The objective of this paper is considerably different from the work cited above. Here we analytically determine the time-optimal pumping strategy for a swing. To do this we first derive the equations of motion for an idealized swing and then apply Pontryagin's Minimum Principle to obtain the necessary conditions for optimality. We then determine the equations for the state and costate vector that satisfy the necessary conditions and subsequently obtain the optimal control law. We shall see that this optimal control law has a very intuitive form.

\section{The Physics of Pumping A Swing}

Observations of children playing on swings show that children who are good at this task all follow a similar strategy. Making the reasonable assumption that the objective is to get as high as possible as quickly as possible, the strategy used by children is to crouch when the swing is at its highest point and to stand-up when the swing passes its lowest point. This is done both on the forward and return cycle. We shall show that this is time-optimal.

To understand the physics behind this pumping we shall make some simplifying assumptions. The swing is ideally modelled as a pendulum. The rider is modelled as a point mass, $m$, and the variable distance of the center of mass of the rider from the fixed support is denoted by $l(t)$. The angle that the swing makes with the vertical is denoted by $\theta(t)$. Conservation of angular momentum for a point mass undergoing planar motion gives, 


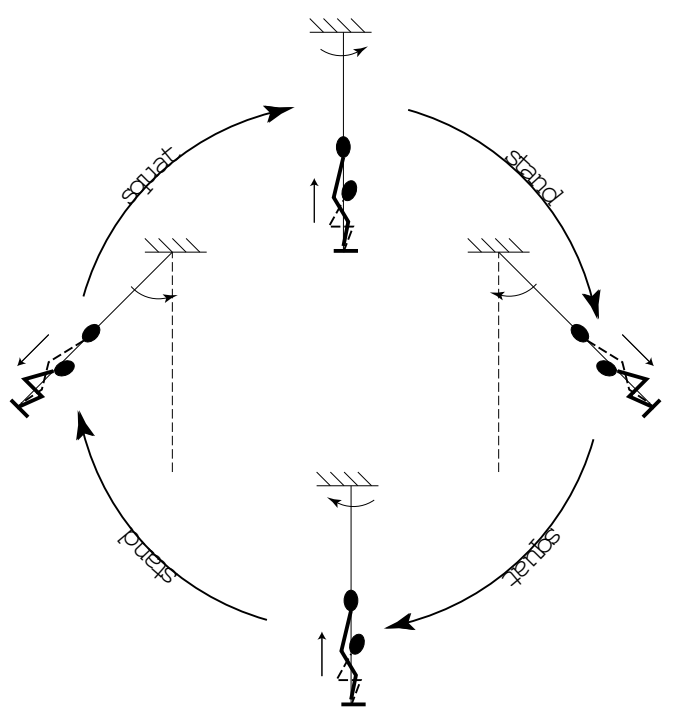

Fig. 1. Pumping strategy to increase the amplitude

$$
\frac{d H}{d t}=\tau,
$$

where $H$ is the angular momentum of the point mass about the fixed support and $\tau$ is the net torque about the fixed support due to all the forces acting on the point mass. Therefore,

$$
\frac{d}{d t}\left(l(t)^{2} \dot{\theta}(t)\right)=-g l(t) \sin (\theta(t)) .
$$

After differentiating and rearranging the terms we get,

$$
\ddot{\theta}+\frac{2 i \dot{\theta}}{l}+\frac{g \sin \theta}{l}=0,
$$

where we have dropped the dependence of $l$ and $\theta$ on time so as to be succinct.

To understand how changing the length of the pendulum can result in an increase or decrease in the amplitude of oscillations, consider first the case when the rider is sitting and is moving towards the origin (Fig. 1). When the rider reaches the origin she instantaneously stand up. In this case, using the fact that, the change in angular momentum is obtained by integrating the torque with respect to time,

$$
l_{\text {stand }}^{2} \dot{\theta}_{\text {stand }}-l_{\text {squat }}^{2} \dot{\theta}_{\text {squat }}=-\int_{t_{0}}^{t_{0}+\delta t} g l \sin (\theta) d t .
$$

Since the process of standing up is carried out instantaneously at $\theta=0$, the right-hand side of (4) evaluates to zero and we have,

$$
\dot{\theta}_{\text {stand }}=\left(\frac{l_{\text {squat }}}{l_{\text {stand }}}\right)^{2} \dot{\theta}_{\text {squat }} .
$$

As $l_{\text {squat }}>l_{\text {stand }}$ the angular velocity of the rider increases by this action and she can attain a larger amplitude of oscillation. Now when the rider reaches the highest point, where the angular velocity is zero, she can again return to the crouching position without losing any angular velocity, as in the ideal case the process is carried out instantaneously. The angle also remains unaffected during this maneuver. The rider is now again in a position to impart another pump to the swing when the swing passes through its lowest point.

The process can be carried out in reverse interchanging the sitting and standing maneuvers and the amplitude of oscillations of the pendulum can be decreased. We thus see how the amplitude of oscillations can be increased or decreased by suitably altering the length of the pendulum.

\section{System EQUATIONS}

Let us now by application of a suitable transformation law convert (3) into a form convenient for the application of Pontryagin's Minimum Principle. We first rewrite (3) as two ordinary differential equations.

$$
\begin{aligned}
& \dot{z}_{1}=z_{2} \\
& \dot{z}_{2}=-\frac{2 i \dot{z}_{1}}{l}-\frac{g \sin \left(z_{1}\right)}{l}
\end{aligned}
$$

Now by making the substitution $x_{2}=z_{2} l^{2}$, we have,

$$
\dot{x}_{2}=-g l \sin \left(z_{1}\right) .
$$

Rewriting $z_{1}$ by $x_{1}$ in (6) we have a set of equations in the variables, $x_{1}$ and $x_{2}$, that capture the dynamics of our system.

$$
\begin{aligned}
& \dot{x}_{1}=\frac{x_{2}}{l^{2}} \\
& \dot{x}_{2}=-g l \sin \left(x_{1}\right) .
\end{aligned}
$$

Let us now make the approximation that, $l(t)=$ $L(1+\epsilon u(t))$, where we have $0<\epsilon \ll 1$ and 
$|u(t)|=1$. We then have after expanding and retaining only the first order terms,

$$
\begin{aligned}
& \dot{x}_{1}=\frac{x_{2}}{L^{2}}-\frac{2 x_{2} \epsilon u(t)}{L^{2}} \\
& \dot{x}_{2}=-g l \sin \left(x_{1}\right)-g l \sin \left(x_{1}\right) \epsilon u(t) .
\end{aligned}
$$

Linearizing about the origin and without any loss of generality setting $L=1$ and $g=1$, we have,

$$
\left[\begin{array}{l}
\dot{x}_{1} \\
\dot{x}_{2}
\end{array}\right]=\left[\begin{array}{cc}
0 & 1 \\
-1 & 0
\end{array}\right]\left[\begin{array}{l}
x_{1} \\
x_{2}
\end{array}\right]-\left[\begin{array}{c}
2 x_{2} \epsilon \\
x_{1} \epsilon
\end{array}\right] u(t)
$$

Or in matrix notational form we have,

$$
\dot{x}(t)=\mathcal{A} x(t)+\mathcal{B}(x(t)) u(t) .
$$

\section{Minimum Time Optimal Control}

\section{A. Statement of Problem}

We shall consider first the problem of bringing a swing from an initial angle to rest in minimum time.

$$
x_{i n i}=\left[\begin{array}{c}
\bar{x}_{1} \\
0
\end{array}\right] \quad x_{f i n}=\left[\begin{array}{l}
0 \\
0
\end{array}\right]
$$

The control input is bounded in magnitude, $|u(t)| \leq 1$.

The problem then, is to determine an optimal input $u^{*}(t)$, such that it satisfies the magnitude bound on the input and takes the state from $x_{i n i}$ to $x_{f i n}$ in minimum time.

We will not go into the theory of optimal control in any great detail other that to state the main result and to apply it to our problem. The interested reader is guided to the excellent reference Athans and Falb (1966). Let us however make a few comments about the problem. First, the system is linear time varying, as can be seen after a slight rearrangement. Also the system is reachable and controllable. The time-optimal control problem is normal, and therefore we can conclude that if a time-optimal control exists for our problem then it is unique. The proofs to support some of our claims can be found in the reference.

\section{B. Pontryagin's Minimum Principle}

Let $u^{*}(t)$ be the time-optimal input and let $x^{*}(t)$ be the corresponding trajectory which satisfies the boundary conditions, i.e. $x^{*}(0)=x_{i n i}$ and $x^{*}\left(T^{*}\right)=x_{\text {fin }}$. Let us define the Hamiltonian $\mathcal{H}$ by the relation,

$$
\begin{aligned}
\mathcal{H}(x(t), p(t), u(t)) & =\lambda_{0} \\
& +\langle p(t), \mathcal{A} x(t)+\mathcal{B}(x(t)) u(t)\rangle .
\end{aligned}
$$

where $\lambda_{0} \geq 0$ and $\langle\cdot, \cdot\rangle$, indicates the usual innerproduct.

Then there exists a corresponding costate vector $p^{*}(t)$ such that,

1)

$$
\begin{gathered}
\dot{x}^{*}(t)=\frac{\partial \mathcal{H}\left(x^{*}(t), p^{*}(t), u^{*}(t)\right)}{\partial p^{*}(t)}, \\
\dot{p}^{*}(t)=-\frac{\partial \mathcal{H}\left(x^{*}(t), p^{*}(t), u^{*}(t)\right)}{\partial x^{*}(t)},
\end{gathered}
$$

with boundary conditions $x^{*}(0)=x_{i n i}$ and $x^{*}\left(T^{*}\right)=x_{\text {fin }}$.

2) We have $\forall t \in\left[0, T^{*}\right]$,

$$
\begin{aligned}
\mathcal{H}\left(x^{*}(t), p^{*}(t), u^{*}(t)\right) & \\
& =\min _{|u(t)| \leq 1} \mathcal{H}\left(x^{*}(t), p^{*}(t), u(t)\right)
\end{aligned}
$$

3) Also $\forall t \in\left[0, T^{*}\right]$ we have,

$$
\mathcal{H}\left(x^{*}(t), p^{*}(t), u^{*}(t)\right)=0 \text {. }
$$

\section{Bang-Bang Control}

From (16) we obtain for our problem that the optimal control, $u^{*}(t)$, is bang-bang. The optimal control is given by,

$$
u^{*}(t)=\operatorname{sgn}\left[2 p_{1}(t) x_{2}(t)+p_{2}(t) x_{1}(t)\right]
$$

Let $\Delta= \pm 1$. Therefore we have,

$$
u^{*}(t)=\Delta= \pm 1
$$




\section{State and Costate vectors}

We have from (14),

$$
\left[\begin{array}{c}
\dot{x}_{1}^{*} \\
\dot{x}_{2}^{*}
\end{array}\right]=\left[\begin{array}{cc}
0 & (1-2 \epsilon \Delta) \\
-(1+\epsilon \Delta) & 0
\end{array}\right]\left[\begin{array}{l}
x_{1}^{*} \\
x_{2}^{*}
\end{array}\right]
$$

with the boundary conditions $x^{*}(0)=x_{i n i}$ and $x^{*}\left(T^{*}\right)=x_{\text {fin }}$.

This can be rewritten as a single second-order differential equation,

$$
\dot{x}(t)+\omega^{2} x(t)=0,
$$

where $\omega=\sqrt{1-\epsilon \Delta-2 \epsilon^{2} \Delta^{2}}$.

Now as $\epsilon \ll 1$ we can make the approximation,

$$
\omega=1-\frac{\epsilon \Delta}{2} .
$$

With this approximation we obtain,

$$
\begin{aligned}
& x_{1}^{*}(t)=x_{i} \cos (\omega t), \\
& x_{2}^{*}(t)=-\frac{\omega x_{i}}{1-2 \epsilon \Delta} \sin (\omega t) .
\end{aligned}
$$

Let us now turn our attention to the costate vector. From (15) we have,

$$
\left[\begin{array}{c}
\dot{p}_{1}^{*} \\
\dot{p}_{2}^{*}
\end{array}\right]=\left[\begin{array}{cc}
0 & (1+\epsilon \Delta) \\
-(1-2 \epsilon \Delta) & 0
\end{array}\right]\left[\begin{array}{l}
p_{1}^{*} \\
p_{2}^{*}
\end{array}\right] .
$$

While we do not have boundary conditions for $p^{*}(t)$ we have from (17),

$\lambda_{0}+x_{2}^{*}(t) p_{1}^{*}\left(1-2 \epsilon u^{*}(t)\right)-x_{1}^{*}(t) p_{2}^{*}\left(1+\epsilon u^{*}(t)\right)=0$.

After eye-balling (24) and (25), we obtain the following equation for $p^{*}(t)$ that will satisfy (25) under certain conditions.

$$
\begin{aligned}
& p_{1}^{*}(t)=\frac{1}{x_{i} \omega} \cos (\omega t+\phi) \\
& p_{2}^{*}(t)=-\frac{1}{x_{i}(1+\epsilon \Delta)} \sin (\omega t+\phi)
\end{aligned}
$$

We now need to determine the condition on $\phi$ so that equations for the state and costate vector satisfy the necessary conditions for optimality. We have using (17),

$$
\lambda_{0}=-\sin (\phi)
$$

As $\lambda_{0} \geq 0$, we have, $-\pi \leq \phi \leq 0$



Fig. 2. Inward Spiral

\section{E. Solution to the time-optimal control problem}

We are now finally in a position to obtain the solution to the optimal control problem. The optimal control input, $u^{*}(t)$, is determined by suitably choosing $\phi$ depending on the boundary conditions on the optimal trajectory, $x^{*}(t)$.

For our problem, with the previously stated boundary conditions, which we repeat here for convenience,

$$
x_{i n i}=\left[\begin{array}{c}
\bar{x}_{1} \\
0
\end{array}\right] \quad x_{f i n}=\left[\begin{array}{l}
0 \\
0
\end{array}\right],
$$

a choice of $\phi=0$ gives us $u^{*}(t)$. We therefore have from (18),

$$
\begin{aligned}
u^{*}(t)= & \operatorname{sgn}\left[2 p_{1}(t) x_{2}(t)+p_{2}(t) x_{1}(t)\right] \\
= & \operatorname{sgn}\left[-\frac{2}{1-2 \epsilon \Delta} \sin (\omega t) \cos (\omega t)\right. \\
& \left.-\frac{1}{1+\epsilon \Delta} \sin (\omega t) \cos (\omega t)\right] \\
= & -\operatorname{sgn}[\sin (\omega t) \cos (\omega t)] \\
= & \operatorname{sgn}\left[x_{1}^{*}(t) x_{2}^{*}(t)\right]
\end{aligned}
$$

We have finally obtained after the application of Pontryagin's Minimum Principle the timeoptimal control law, which states that the controller switches between +1 and -1 when one of the states goes to zero. This corresponds to our initial observation regarding the pumping of a swing. 


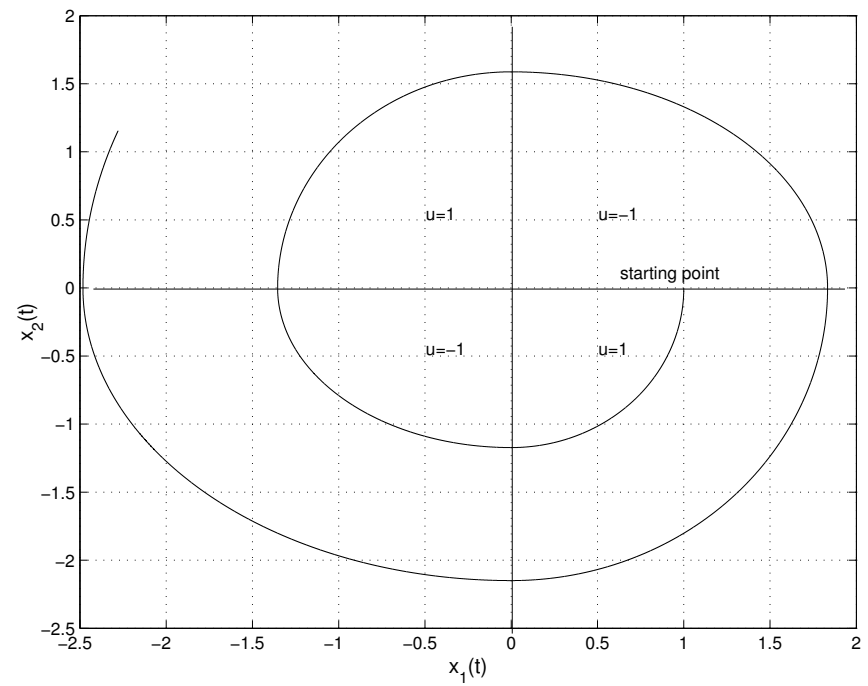

Fig. 3. Outward Spiral

The case when we start with small initial oscillations and try to move away from the origin in minimal time mimics the development above with the difference being that we obtain, $u^{*}(t)=$ $-\operatorname{sgn}\left[x_{1}^{*}(t) x_{2}^{*}(t)\right]$. Fig. 2 and Fig. 3 show the trajectories in the phase space for the two cases discussed.

\section{Conclusion}

Using the Minimum Principle and after some simplifications we have proved that the pumping strategy used by children on swings is timeoptimal. The observation that it is time-optimal appeals to our intuition, as children playing on a swing would not try to optimize criterion like energy, but rather would try to have as much fun as possible, which would in general mean that they try to get as high as possible as quickly as possible. We should point out that the result has been obtained after making linearizing assumptions and as such is valid as long as that the assumptions are valid. We should also note that, while we have considered the cases $\phi=-\pi$ and $\phi=0$, other values for $\phi$ have to be chosen for different boundary conditions.

\section{ACKNOWLEDGEMENTS}

I would like to thank Prof Andy Ruina, Tama's Kalmar-Nagy, Matthew Earl, and Cedric Langbort, all from the Department of Theoretical and Applied Mechanics at Cornell University for many interesting discussion regarding this and related problems.

\section{REFERENCES}

[1] M. Athans and P. L. Falb. Optimal Control. McGraw-Hill Book Co., New York, 1966.

[2] J. E. A. Bertram and A. Ruina. Multiple walking speed-frequency relations are predicted by constrained optimization. Journal of Theoretical Biology, 209:445-453, 2001.

[3] J. A. Burns. More on pumping a swing. American Journal of Physics, 38:920-922, 1970.

[4] M. G. Pandy and F. C. Anderson. Dynamic simulation of human movement using largescale models of the body. Phonetica, 57:219228, 2000.

[5] P. L. Tea and H. Falk. Pumping on a swing. American Journal of Physics, 36:1165-1166, 1968.

[6] S. Wirkus, R. Rand, and A. Ruina. How to pump a swing. College Mathematics Journal, 29(4):266-275, 1998. 